\title{
Boundary layer flow of magneto-micropolar nanofluid flow with Hall and ion-slip effects using variable thermal diffusivity
}

\author{
M. BILAL*, S. HUSSAIN, and M. SAGHEER \\ Department of Mathematics, Capital University of Science and Technology, Islamabad, Pakistan
}

\begin{abstract}
In the present article, magneto-micropolar nanofluid flow with suction or injection in a porous medium over a stretching sheet for the heat and mass transfer is analyzed numerically. Both Hall and ion-slip effects are considered along with variable thermal diffusivity. The governing partial differential equations are transformed to ordinary differential equations using usual similarity transformations. These coupled non-linear differential equations are solved using the shooting method. Effects of prominent parameter on velocities, temperature and concentration are discussed graphically. Numerical values of skin-friction coefficient, local Nusselt number and local Sherwood number are also tabulated and discussed.
\end{abstract}

Key words: Hall effect, nanofluid, ion-slip effect, magneto-micropolar fluid, shooting method.

$\begin{array}{ll}\text { Nomenclature } \\ b & \text { constants of dimension (time) }{ }^{-1} \\ B & \text { magnetic induction vector } \\ B_{0} & \text { magnetic field } \\ \mathrm{C} & \text { concentration of fluid } \\ C f_{x} & \text { skin friction along x-axis } \\ C_{z} & \text { skin friction along z-axis } \\ C_{w} & \text { concentration at wall } \\ C_{\infty} & \text { ambient concentration } \\ c_{p} & \text { specific heat } \\ D_{B} & \text { Brownian diffusion coefficient } \\ D_{T} & \text { thermophoretic diffusion coeff. } \\ E & \text { electric field intensity } \\ e & \text { charge of an electron } \\ E c & \text { Eckert number } \\ f, g & \text { dimensionless stream functions } \\ f_{w} & \text { mass transfer coefficient } \\ G & \text { microrotation parameter } \\ G_{1} & \text { spin gradient viscosity }\end{array}$

$\begin{array}{ll}h & \text { microrotation function } \\ J & \text { electric current vector } \\ K & \text { vortex velocity } \\ \kappa_{p} & \text { permeability parameter } \\ k^{*} & \text { permeability of porous medium } \\ L e & \text { Lewis number } \\ M & \text { magnetic parameter } \\ N & \text { microrotation component } \\ N_{b} & \text { Brownian motion parameter } \\ N_{t} & \text { thermophoresis parameter } \\ N_{1} & \text { coupling constant parameter } \\ n_{e} & \text { density number of electrons } \\ P_{r} & \text { Prandtl number } \\ R_{e} & \text { Reynolds number } \\ S h_{x} & \text { Sherwood number } \\ T & \text { temperature of fluid } \\ T_{w} & \text { temperature at wall } \\ T_{\infty} & \text { ambient temperature } \\ U_{s} & \text { surface velocity } \\ V & \text { velocity vector }\end{array}$

$\begin{array}{ll}V_{w} & \text { suction injection velocity } \\ (u, v, w) & \text { velocity components } \\ (x, y) & \text { coordinate axises } \\ \alpha & \text { thermal diffusivity } \\ \alpha_{0} & \text { thermal diffusivity at wall temperature } \\ \beta_{2} & \text { fluid nature dependent parameter } \\ \beta_{e} & \text { Hall current parameter } \\ \beta_{i} & \text { ion-slip parameter } \\ v & \text { kinematic viscosity } \\ v_{e} & \text { electron atom collision frequency } \\ \theta & \text { dimensionless temperature } \\ \phi & \text { dimensionless concentration } \\ \sigma & \text { electric conductivity } \\ \omega & \text { collision frequency } \\ \rho & \text { density of fluid } \\ \tau_{w} & \text { wall shear stress } \\ \tau_{z} & \text { shear stress } \\ \eta & \text { dimensionless variable } \\ \mu & \text { dynamic viscosity }\end{array}$

\section{Introduction}

The idea of a nanofluid was first introduced by Choi [1] in 1995. While using the nanofluids, the maximum possible thermal properties are targeted to be achieved with the minimum possible concentration by uniform dispersion and stable suspension of nanoparticles in the host fluids [2, 3]. These fluids are capable of increasing the thermophysical properties, such as thermal diffusivity, thermal conductivity, viscosity, and convective heat transfer coefficient, as compared to those

\footnotetext{
*e-mail: me.bilal.786@gmail.com
}

of base fluids like water, ethylene, or triethylene glucose and other coolants, biofluids, and polymer solutions, as elaborated by Choi [4] and Wong [5]. These fluids possess the unique physical and chemical properties and can easily pass through the microchannels and capillaries and don't block the flow. Because of these properties, nanofluids are now extensively used in different industries, engineering and biomedical sectors. These can be used in automotives, nuclear reactors, transportation, power generation, thermal therapy for cancer treatment, micromanufacturing, metallurgical, and chemical sectors [5]. Buongiorno [6] considered Brownian diffusion and thermophoresis slip mechanism for the relative velocity of base fluid and nanoparticles. A subbranch of nanofluids termed magnetic 
nanofluids has also shown significant contribution to numerous engineering fields [7]. These fluids respond to the applied magnetic fields and allow for further manipulation of heat transfer and hydrodynamic characteristics. Magnetite and aluminum oxide nanoparticles are often employed in the architecture of such fluids. Shehzad et al. [8] worked on mixed convection flow of a Maxwell nanofluid with the doubly-stratified heat generation/absorption. Magnetohydrodynamics stagnation point flow of a nanofluid over an exponentially stretching sheet with an effect of chemical reaction, heat source, and suction/injunction was discussed by Reddy et al. [9]. Mansur et al. [10] studied the magnetohydrodynamic stagnation point flow of a nanofluid over a stretching/shrinking sheet with suction. Biomedical uses for magnetic nanofluids have been confirmed by Patel [11]. Yadav et al. [12] worked on magneto nanolfuid flow with the Hall current effect. For recent research related to nanofluids, [13-17] can be consulted.

A lot of research work related to MHD Micropolar fluid over a stretching sheet has been explained under different physical effects. Most often, Hall current and ion-slip effects are neglected because of their ignorable contribution while applying Ohm's law for small magnetohydrodynamics [18]. However, these have a strong effect when the magnetic field is high [19], due to the strong electromagnetic force. Hall effect plays an important role when the Hall parameter is high. Hall parameter is the ratio of electron cyclotron frequency to atom-electron collision frequency. The Hall current effect is high when the electron-atom collision frequency is low [18]. It is also known that the mass of ions is much greater than the mass of electrons, and ultimately their motion will be different from each other. As a result of different velocities of electrons and ions, their diffusion velocities will also be different. As the current density mainly depends upon the diffusion velocity, the current density of electrons is much higher than that of ions. However, if the magnetic field is high, then the diffusion velocity of ions is not negligible. The combined effect of diffusion velocities of ions and electrons is called the ion-slip effect. Hall and ion-slip effects are involved in many engineering technologies, such as Hall sensors, Hall accelerators, construction of centrifugal pumps and turbines, etc. Attia et al. [20] discussed the heat transfer of Couette flow of a dusty fluid with ion-slip effect and uniform suction and injection. MHD mass transfer problem by free convection flow of an ionized incompressible viscous micropolar fluid across an infinite vertical plate under the action of Hall current and ion-slip parameter has been discussed by Anika et al. [21]. Ziya Uddin et al. [22] considered the Hall and ion-slip effect on MHD boundary layer flow of a micropolar fluid past a wedge. Motsa et al. [23] studied Hall and ion-slip effect for the micropolar fluid flow with chemical reaction and thermal diffusivity.

Eringen [24, 25] put forth a theory of micropolar fluids based on the characteristics of inertial properties of structure particles which undergo rotation. The microscopic effects and micro motion of structure fluid elements describe the micropolar fluids. In mathematical modeling, the interaction of microrotation field and macro velocity field can be portrayed by new material constant in classical Newtonian fluids. Eringen's fluid model, based on classical Navier-Stokes equations, is a special

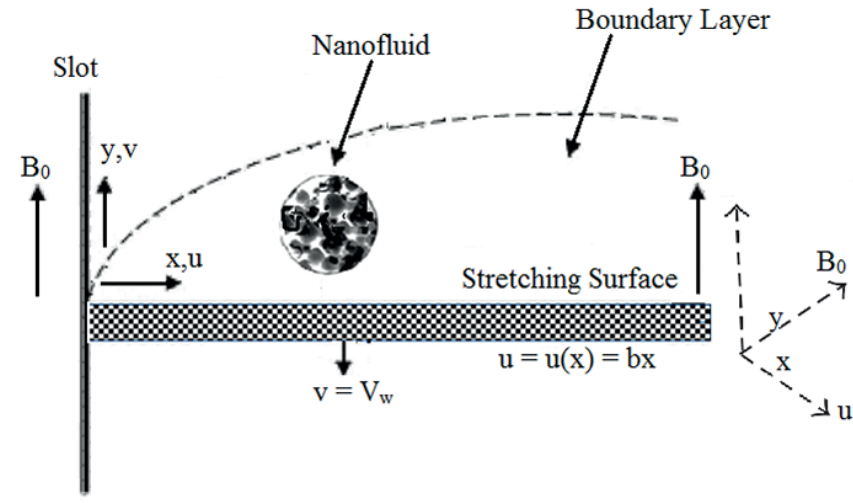

Fig. 1. Geometry of the problem

case for incompressible and viscous fluids. These models are also suitable for the study of colloidal fluids flow, polymers, lubricants, cerebro fluids, liquid crystals, animal blood, real fluids with suspensions, ferro fluids, etc., for which the classical Navier-Stokes theory is inadequate. In the micropolar fluid equation, the gyration parameter and microrotation vectors appear along with classical Navier-Stokes equations. Realising the significance and application of micropolar fluids, many other researchers have also worked in this area [26-31].

The aim of the present study is to examine the effect of nanofluid on heat and mass transfer with the impact of Hall current and ion-slip effects on an electrically conducting magneto-micropolar fluid flowing on a linearly stretched sheet. Generally, there are two categories of simulation of nanofluid convective heat transfer modeling: single phase and double phase modeling. In single phase modeling, the combination of base fluid and nanoparticles is considered a homogenous mixture and their properties are studied collectively, whereas in the two phase model, the behaviour and properties of nanoparticles are considered separately from the properties and behaviour of the base fluid. In the present study, a single phase model has been considered. A variable thermal diffusivity is considered in the formation of energy equation. The dynamics of the flow are described by the conservation laws of mass, momentum, energy, and concentration. The governing set of nonlinear PDEs are reduced into a system of ODEs, and then solved numerically through the shooting technique. The quantities such as velocity, concentration, temperature, Nusselt number, Sherwood number, and skin-friction for the variation of emerging parameters are computed and elaborated. To the best of our knowledge, the study of Hall current and ion-slip effect with variable thermal diffusivity and magneto-micropolar nanofluids has not been discussed in literature yet.

\section{Mathematical formulation}

A steady, viscous, and incompressible, electrically conducting magneto-micropolar nanofluid flow over a horizontal, porous plate stretching linearly with velocity $u_{x}$ along the $x$-axis 
through a porous medium has been considered. A strong constant magnetic field of intensity $B_{0}$ in the direction along $y$-axis is also assumed. Due to this strong magnetic field strength, the electrically conducting fluid has the Hall and ion-slip effects, which give rise to a force on the fluid in the $z$-direction, and hence the flow becomes three-dimensional.

Elgazery [32] generalized the Ohm's law for the Hall current in the form:

$$
\mathbf{J}=\frac{\sigma}{1+\left(\omega / v_{e}\right)^{2}}\left(\mathbf{E}+(\mathbf{V} \times \mathbf{B})-\frac{1}{e n_{e}}(\mathbf{J} \times \mathbf{B})\right),
$$

where $\omega$ is the electron cyclotron and $v_{e}$ is the electron-atom collision frequency. When the ratio $\omega / v_{e}$ is very large, the phenomenon is called "ion-slip". By considering all the fluid property constants, except for thermal diffusivity, and applying Boussinesq approximations, the governing equations for the considered problem become:

$$
\begin{aligned}
\frac{\partial u}{\partial x}+\frac{\partial v}{\partial y}= & , \\
u \frac{\partial u}{\partial x}+v \frac{\partial u}{\partial y} & =v \frac{\partial^{2} u}{\partial y^{2}}+\frac{K}{\rho} \frac{\partial N}{\partial y}-\frac{\mu}{\rho k^{*}} u \\
& -\frac{\sigma B_{0}^{2}}{\rho\left(\alpha_{e}^{2}+\beta_{e}^{2}\right)}\left(\alpha_{e} u+\beta_{e} w\right),
\end{aligned}
$$

$$
\begin{aligned}
& u \frac{\partial w}{\partial x}+v \frac{\partial w}{\partial y}=v \frac{\partial^{2} w}{\partial y^{2}}+\frac{\sigma B_{0}^{2}}{\rho\left(\alpha_{e}^{2}+\beta_{e}^{2}\right)} \\
& \left(\beta_{e} u-\alpha_{e} w\right)-\frac{\mu}{\rho k^{*}} w,
\end{aligned}
$$

$$
\frac{G_{1}}{K} \frac{\partial^{2} N}{\partial y^{2}}-2 N-\frac{\partial u}{\partial y}=0
$$

$$
\begin{aligned}
u \frac{\partial T}{\partial x} & +v \frac{\partial T}{\partial y}=\frac{\partial}{\partial y}\left(\alpha \frac{\partial T}{\partial y}\right)+\frac{\mu}{\rho c_{p}}\left[\left(\frac{\partial u}{\partial y}\right)^{2}\right. \\
& \left.+\left(\frac{\partial w}{\partial y}\right)^{2}\right]+\frac{1}{\rho c_{p}} \frac{\sigma B_{0}^{2}}{\left(\alpha_{e}^{2}+\beta_{e}^{2}\right)}\left(u^{2}+w^{2}\right) \\
& +\tau\left[D_{B}\left(\frac{\partial T}{\partial y} \frac{\partial C}{\partial y}\right)+\frac{D_{T}}{T_{\infty}}\left(\frac{\partial T}{\partial y}\right)^{2}\right]
\end{aligned}
$$$$
u \frac{\partial C}{\partial x}+v \frac{\partial C}{\partial y}=D_{B} \frac{\partial^{2} C}{\partial y^{2}}+\frac{D_{T}}{T_{\infty}} \frac{\partial^{2} T}{\partial y^{2}} .
$$

where the last terms of (3) and (4) appear due to the porosity of the medium, $D_{B}$ and $D_{T}$ are thermodiffusion and thermophoresis terms, respectively, and $\partial N / \partial y$ in (3) represents the micropolar effects of particles. The corresponding boundary conditions for the governing PDEs are as follows:

$$
\begin{aligned}
& \text { At } y=0: u=u_{x}=b x, \quad v=-V_{w}, \quad N=0, \\
& \qquad \begin{array}{l}
w=0, \quad T=T_{w}, \quad C=C_{w}, \\
\text { As } y \rightarrow \infty: u \rightarrow 0, \quad w \rightarrow 0, \quad N \rightarrow 0, \\
\quad T \rightarrow T_{\infty}, \quad C \rightarrow C_{\infty} .
\end{array}
\end{aligned}
$$

Here, $V_{w}$ is the velocity of suction $(>0)$ or injection $(<0)$, $\alpha_{e}=1+\beta_{i} \beta_{e}$, where $\beta_{i}$ and $\beta_{e}$ are ion-slip and Hall current parameters, respectively, $b$ is a dimensional constant with the unit $(\text { time })^{-1}$.

The dependence of thermal diffusivity on temperature is given by

$$
\alpha=\alpha_{0}\left(1+\beta_{2} \theta\right)
$$

The similarity transformations which are used to convert the partial differential equations into ordinary differential equations are frequently used in many research articles $[43,44]$

$$
\begin{aligned}
& \eta=\sqrt{\frac{b}{v}} y, \quad u=b x f^{\prime}(\eta), \quad v=-\sqrt{b v} f(\eta), \\
& w=\sqrt{b v} g(\eta), \quad N=\sqrt{\frac{b^{3}}{v}} x h(\eta), \\
& \theta(\eta)=\frac{T-T_{\infty}}{T_{w}-T_{\infty}}, \quad \phi(\eta)=\frac{C-C_{\infty}}{C_{w}-C_{\infty}} .
\end{aligned}
$$

(2) is satisfied identically, while the resulting ordinary differential equations are:

$$
\begin{gathered}
f^{\prime \prime \prime}+f f^{\prime \prime}-\left(f^{\prime}\right)^{2}+N_{1} h^{\prime}-\frac{1}{k_{p}} f^{\prime} \\
-\frac{M}{\left(\alpha_{e}^{2}+\beta_{e}^{2}\right)}\left(\alpha_{e} f^{\prime}+\frac{\beta_{e}}{\sqrt{R e}} g\right)=0 \\
g^{\prime \prime}+f g^{\prime}+\frac{M}{\left(\alpha_{e}^{2}+\beta_{e}^{2}\right)}\left(\beta_{e} \sqrt{\operatorname{Re}} f^{\prime}-\alpha_{e} g\right)-\frac{1}{k_{p}} g=0, \\
\theta^{\prime \prime}+\frac{\beta_{2}}{1+\beta_{2} \theta} \theta^{\prime 2}+\frac{\operatorname{Pr}}{1+\beta_{2} \theta} f \theta^{\prime \prime}+2 h-f^{\prime \prime}=0 \\
+\frac{\operatorname{Pr}^{\prime} b}{1+\beta_{2} \theta}\left(\theta^{\prime} \phi^{\prime}+\frac{N t}{N b} \theta^{\prime 2}\right)+\frac{\operatorname{PrEc}}{1+\beta_{2} \theta}\left[f^{\prime \prime 2}+\right. \\
\left.+\frac{g^{\prime 2}}{\operatorname{Re}}+\frac{M}{\left(\alpha_{e}^{2}+\beta_{e}^{2}\right)}\left(f^{\prime 2}+\frac{g^{2}}{\operatorname{Re}}\right)\right]=0,
\end{gathered}
$$


The transformed boundary conditions are:

$$
\begin{aligned}
& f(0)=f_{w}, \quad f^{\prime}(0)=1, \quad g(0)=0, \quad h(0)=0, \\
& \theta(0)=1, \quad \phi(0)=1, \quad f^{\prime}(\infty)=0, \quad g(\infty)=0 \\
& h(\infty)=0, \quad \theta(\infty)=0, \quad \phi(\infty)=0 .
\end{aligned}
$$

Different dimensionless parameters appearing in (11-16) are:

$$
\begin{aligned}
& N_{1}=\frac{K}{\rho v}, M=\frac{\sigma B_{0}^{2}}{\rho b}, G=\frac{G_{1} b}{K v}, \operatorname{Pr}=\frac{v}{\alpha}, \\
& f_{w}=\frac{V_{w}}{\sqrt{b v}}, E c=\frac{(b x)^{2}}{c_{p}\left(T_{w}-T_{\infty}\right)}, k_{p}=\frac{k^{*} b \rho}{\mu}, \\
& R e=\frac{b x^{2}}{v}, \gamma=\frac{k v}{(b x)^{2}}, N b=\frac{\tau D_{B}}{v}\left(C_{w}-C_{\infty}\right), \\
& N t=\frac{D_{T}}{T_{\infty}} \frac{\tau}{v}\left(T_{w}-T_{\infty}\right), L e=\frac{\alpha}{D_{B}} .
\end{aligned}
$$

The important quantities of interest, such as the coefficient of skin friction in $x$ and $z$ directions, local Nusselt number, and Sherwood number are defined as:

$$
\begin{aligned}
C f_{x} & =\frac{\tau_{w}}{\rho b x \sqrt{b v}}, C f_{z}=\frac{\tau_{z}}{\rho b x \sqrt{b v}}, \\
N u_{x} & =\frac{-\left(\frac{\partial T}{\partial y}\right)_{y=0}}{\left(T_{w}-T_{\infty}\right) \sqrt{\frac{b}{v}}}, S h_{x}=\frac{-\left(\frac{\partial C}{\partial y}\right)_{y=0}}{\left(C_{w}-C_{\infty}\right) \sqrt{\frac{b}{v}}} .
\end{aligned}
$$

Here, the local wall shear stresses are defined as:

$$
\begin{aligned}
& \tau_{w}=\left[(\mu+K)\left(\frac{\partial u}{\partial y}\right)+K N\right]_{y=0}, \\
& \tau_{z}=\left[(\mu+K)\left(\frac{\partial w}{\partial y}\right)\right]_{y=0} .
\end{aligned}
$$

The dimensionless form of skin friction, local Nusselt number, and Sherwood number is:

$$
\begin{aligned}
& C f_{x}=\left(1+N_{1}\right) f^{\prime \prime}(0), C f_{z}=\left(1+N_{1}\right) g^{\prime}(0), \\
& N u_{x}=-\theta^{\prime}(0), S h_{x}=-\phi^{\prime}(0) .
\end{aligned}
$$

\section{Solution methodology}

The resulting system of nonlinear ODEs (11-15) subject to the conditions (16) has been explored numerically through the shooting method [33], which is used frequently by many researchers to obtain solutions for such types of problems [34-38] for various values of different parameters. To apply the shooting method, the system of nonlinear ODEs (11-15) is converted to the following system of first-order ODEs:

$$
\begin{aligned}
& y_{1}^{\prime}=y_{2} \text {, } \\
& y_{1}(0)=f_{w} \\
& y_{2}^{\prime}=y_{3} \text {, } \\
& y_{3}^{\prime}=y_{2}^{2}-y_{1} y_{3}-N_{1} y_{7}+\frac{1}{k_{p}} y_{2} \\
& y_{2}(0)=1 \\
& +\frac{M}{\alpha_{e}^{2}+\beta_{e}^{2}}\left(\alpha_{e} y_{2}+\frac{\beta_{e}}{\sqrt{R e}} y_{4}\right) \\
& y_{3}(0)=p \\
& y_{4}^{\prime}=y_{5} \text {, } \\
& y_{5}^{\prime}=y_{1} y_{5}-\frac{M}{\alpha_{e}^{2}+\beta_{e}^{2}}\left(\beta_{e} \sqrt{\operatorname{Re}} y_{2}-\alpha_{e} y_{4}\right) \\
& y_{4}(0)=0 \\
& +\frac{1}{k_{p}} y_{4}, \\
& y_{5}(0)=q \\
& y_{6}^{\prime}=y_{7} \text {, } \\
& y_{7}^{\prime}=\frac{1}{G}\left(2 y_{6}+y_{3}\right) \text {, } \\
& y_{6}(0)=0 \\
& y_{8}^{\prime}=y_{9} \text {, } \\
& y_{7}(0)=r \\
& y_{9}^{\prime}=\frac{-1}{\beta_{2} y_{8}+1}\left[\begin{array}{l}
\beta_{2} y_{9}^{2}+\operatorname{PrEc}\left(y_{3}^{2}+\frac{1}{\operatorname{Re}} y_{5}^{2}\right)+ \\
\frac{\operatorname{PrEcM}}{\alpha_{e}^{2}+\beta_{e}^{2}}\left(y_{2}^{2}+\frac{1}{\operatorname{Re}} y_{4}^{2}\right)+\operatorname{Pr}_{1} y_{9}
\end{array}\right] \\
& y_{8}(0)=1 \\
& +\frac{P r N b}{1+\beta_{2} y_{8}}\left(y_{8} y_{11}+\frac{N t}{N b} y_{9}^{2}\right) \text {, } \\
& y_{10}(0)=1 \\
& y_{11}^{\prime}=-L e P r y_{1} y_{11}-\frac{N t}{N b} y_{9}^{\prime} \text {. } \\
& y_{11}(0)=t
\end{aligned}
$$

Here, $f$ is denoted by $y_{1}, g$ by $y_{4}, h$ by $y_{6}, \theta$ by $y_{8}$, and $\phi$ by $y_{10}$. After choosing the five missing conditions, the above system of first-order ODEs is solved by using the Runge-Kutta method of order four. To refine the missing initial conditions $p, q, r, s$, and $t$, the Newton's iterative scheme is used, requiring another system of 55 first-order ODEs along with the initial conditions. This new system is then solved using the RK-4 method. The solution of this IVP is then used to construct the Jacobian matrix involved in the Newton's iterative method. On the basis of a number of computational experiments, we are considering $[0,8]$ as the domain of the problem, instead of $[0, \infty]$. The stopping criteria for the iterative process is set as:

$$
\begin{gathered}
\max \left\{\left|y_{2}(8)\right|,\left|y_{4}(8)\right|,\left|y_{6}(8)\right|,\right. \\
\left.\left|y_{8}(8)\right|,\left|y_{10}(8)\right|\right\}<\varepsilon .
\end{gathered}
$$

All the computations are performed with the tolerance of $\varepsilon=10^{-8}$, using a verified Matlab code. To strengthen the results obtained by the shooting method, we have also solved this system of ODEs with MATLAB's built-in function bvp4c. It is a finite difference code that implements the three-stage Lobatto IIIa formula. This is a collocation formula, and the collocation polynomial provides a $C^{1}$-continuous solution that is fourthorder accurate, uniformly in $[\mathrm{a}, \mathrm{b}]$. Mesh selection and error control are based on the residual of the continuous solution. It has the following Matlab syntax:sol=bvp4c(@odefun,@ bcfun, solinit, options). Further details can be found in [39]. Many researchers used this package to solve their BVPs, see for example [40-42]. 


\section{Results and discussion}

This section is devoted to the detailed discussion of the numerical solutions of our problem. In Table 1, a comparison between the present results, obtained using the shooting method and bvp4c in the absence of a nanofluid, and those given by Motsa et al. [23], has been presented. An excellent agreement is observed between these results, which strengthens our methodology. To see the effect of physical parameter on skin-friction coefficient, local Nusselt and Sherwood number, numerical results are obtained and tabulated.

Table 1

Comparison of the present results with those of Motsa for $R e=1.0$, $G=2.0, k_{p}=2.0, f_{w}=0.1, \operatorname{Pr}=0.72, E c=0.02, L e=0.014$ and

$$
\beta_{2}=0.5
$$

\begin{tabular}{|c|c|c|c|c|c|c|c|c|c|}
\hline \multirow[b]{3}{*}{$M$} & \multirow[b]{3}{*}{$\beta_{e}$} & \multirow[b]{3}{*}{$\beta_{i}$} & \multirow[b]{3}{*}{$N_{1}$} & \multicolumn{3}{|c|}{$-\left(1+N_{1}\right) f^{\prime \prime}(0)$} & \multicolumn{3}{|c|}{$\left(1+N_{1}\right) g^{\prime}(0)$} \\
\hline & & & & \multirow[t]{2}{*}{ Motsa } & \multicolumn{2}{|l|}{ Present } & \multirow[t]{2}{*}{ Motsa } & \multicolumn{2}{|l|}{ Present } \\
\hline & & & & & shooting & bvp $4 c$ & & shooting & bvp $4 c$ \\
\hline 1 & 5 & 0.4 & 0.2 & 1.563229 & 1.563229 & 1.563228 & 0.085991 & 0.085989 & 0.085991 \\
\hline 2 & & & & 1.610774 & 1.610774 & 1.610774 & 0.164225 & 0.164221 & 0.164226 \\
\hline 3 & & & & 1.659723 & 1.659724 & 1.659723 & 0.235543 & 0.235536 & 0.235543 \\
\hline 4 & & & & 1.709225 & 1.709227 & 1.709225 & 0.300892 & 0.300882 & 0.300892 \\
\hline 5 & & & & 1.758728 & 1.758731 & 1.758728 & 0.361156 & 0.361142 & 0.361156 \\
\hline 0.3 & 0 & & & 1.658124 & 1.658124 & 1.658124 & 0 & 0 & 0 \\
\hline & 2 & & & 1.555081 & 1.555081 & 1.555080 & 0.048859 & 0.048859 & 0.048860 \\
\hline & 4 & & & 1.535302 & 1.535302 & 1.535301 & 0.031718 & 0.031718 & 0.031719 \\
\hline & 6 & & & 1.528942 & 1.528941 & 1.528941 & 0.022919 & 0.022919 & 0.022920 \\
\hline & 5 & 0 & & 1.524377 & 1.524377 & 1.524376 & 0.035104 & 0.035104 & 0.035105 \\
\hline & & 0.5 & & 1.532225 & 1.532225 & 1.532224 & 0.024303 & 0.024302 & 0.024303 \\
\hline & & 1 & & 1.532737 & 1.532737 & 1.532736 & 0.014833 & 0.014832 & 0.014833 \\
\hline & & 1.5 & & 1.531103 & 1.531103 & 1.531102 & 0.009319 & 0.009319 & 0.009319 \\
\hline & & 0.4 & 0 & 1.287381 & 1.286722 & 1.286722 & 0.020385 & 0.022352 & 0.022352 \\
\hline & & & 0.2 & 1.532225 & 1.531435 & 1.531435 & 0.024303 & 0.026647 & 0.026648 \\
\hline & & & 0.5 & 1.891325 & 1.890341 & 1.890339 & 0.030062 & 0.032963 & 0.032963 \\
\hline & & & 1.0 & 2.467535 & 2.466230 & 2.466226 & 0.039313 & 0.043105 & 0.043106 \\
\hline
\end{tabular}

Table 2

Numerical values of $C f_{x}, C f_{z}$, when $R e=1.0, G=0.8, L e=2.0$, $E c=0.02, \operatorname{Pr}=0.72, N b=0.3, N t=0.7, \beta_{2}=0.8$

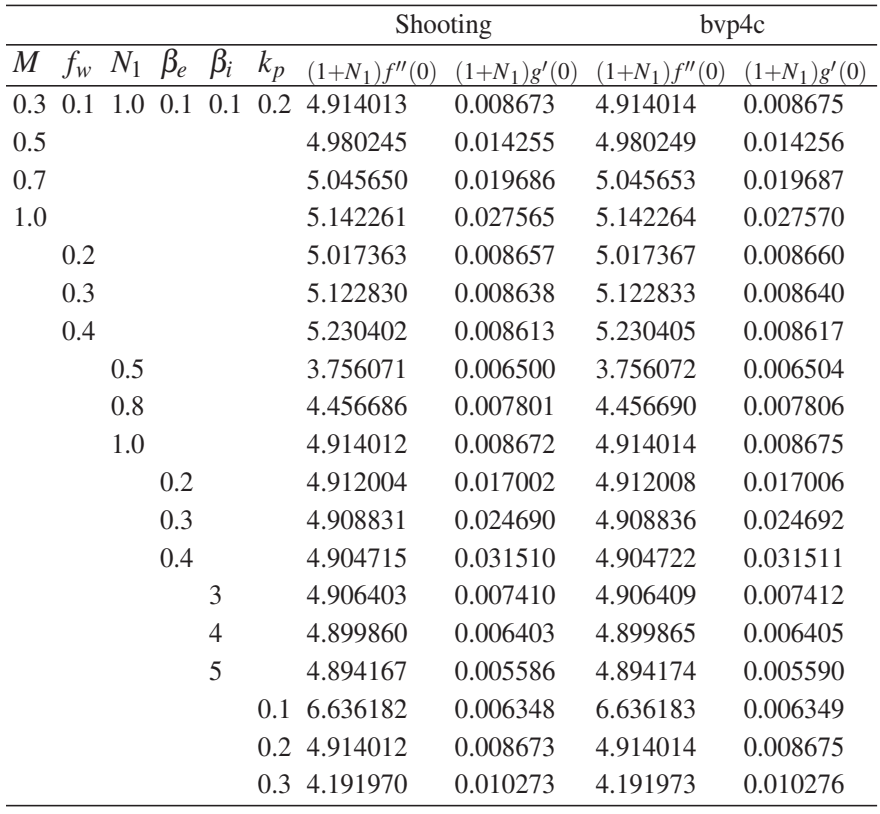

Table 3

Numerical values of $N u_{x}$ and $S h_{x}$ for various values of $\operatorname{Pr}, f_{w}, k_{p}, R e$, $G, E c, M, \beta_{e}, \beta_{i}, N_{1}$

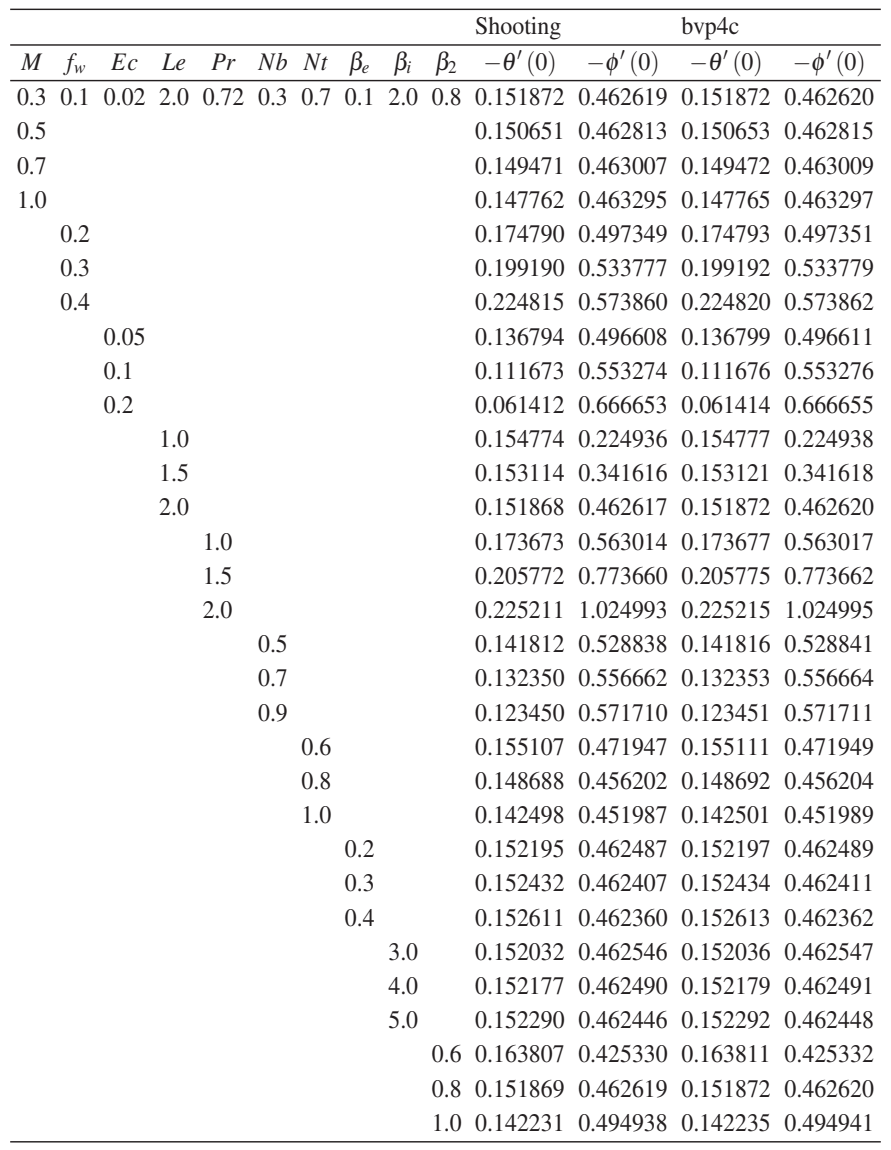

From Table 2, it is noticed that the skin friction coefficient $C f_{x}$ in the $x$ direction increases with the increasing value of magnetic parameter $M$, coupling parameter $N_{1}$, and mass transfer parameter $f_{w}$, whereas it is a decreasing function for increasing values of $\beta_{e}, \beta_{i}$, and $k_{p}$. Table 2 also shows the variation of parameters for skin friction coefficient in the $z$ direction. It is observed that $C f_{z}$ increases with the increase of magnetic parameter $M$, material parameter $N_{1}$, Hall current parameter $\beta_{e}$, and permeability parameter $k_{p}$. However, by increasing $f_{w}$ and $\beta_{i}$, the skin friction decreases. In Table 3, the effect of magnetic parameter $M$, mass transfer parameter $f_{w}$, Eckert number $E c$, Lewis number $L e$, Brownian motion parameter $N b$, thermophoresis parameter $N t$, Hall current parameter $\beta_{e}$, ion-slip parameter $\beta_{i}$, variable thermal diffusivity parameter $\beta_{2}$, and Prandtl number $\operatorname{Pr}$ on local Nusselt number and Sherwood number are shown. From the table, it is deducted that $M, E c, L e, N b, N t$, and $\beta_{2}$ have a reductive effect on the Nusselt number, which in turn increases for the increasing values of $f_{w}, \operatorname{Pr}, \beta_{e}$, and $\beta_{i}$. Furthermore, the magnitude of local Sherwood number $-\phi^{\prime}(0)$ increases when $M, f_{w}, E c, L e, P r, N b$, and $\beta_{2}$ are increased, while it decreases with an increase of $\beta_{i}, \beta_{e}$, and $N t$.

To visualize the effect of different physical parameters on tangential velocity $f^{\prime}(\eta)$, lateral velocity $g(\eta)$, angular velocity $h(\eta)$, temperature $\theta(\eta)$, and nanoparticle concentration profiles 
$\phi(\eta)$, Figs. 2-9 are plotted. Fig. 2 depicts the effect of Hall parameter $\beta_{e}$ on velocity components along $x$ and $z$ directions respectively. The inclusion of Hall parameter decreases the resistive force imposed by the magnetic field due to its effect of reducing the effective conductivity. Hence, the velocity component increases as the Hall parameter increases, but this increase in velocity is very small. Similarly, the transverse velocity also increases with the increasing value of $\beta_{e}$. The temperature distribution $\theta(\eta)$ increases by increasing the variable thermal diffusivity parameter $\beta_{2}$, and this fact is shown in Fig. 3. In Fig. 3, the effect of permeability parameter $k_{p}$ on velocity $f^{\prime}(\eta)$ profile is shown. From the figure, it is clear that stream velocity $f^{\prime}(\eta)$
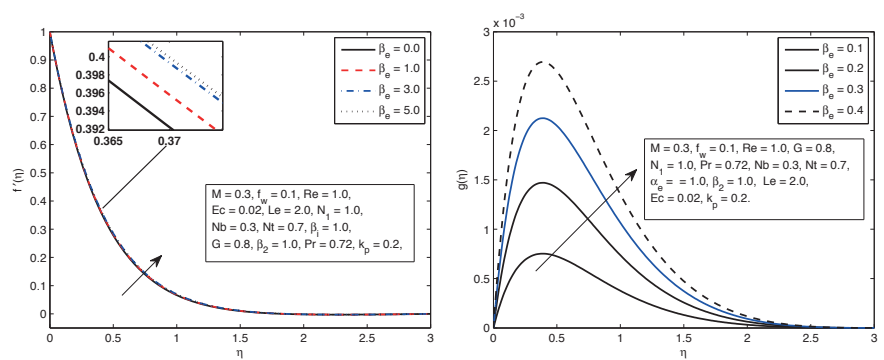

Fig. 2. Influence of $\beta_{e}$ on $f^{\prime}$ and $g$
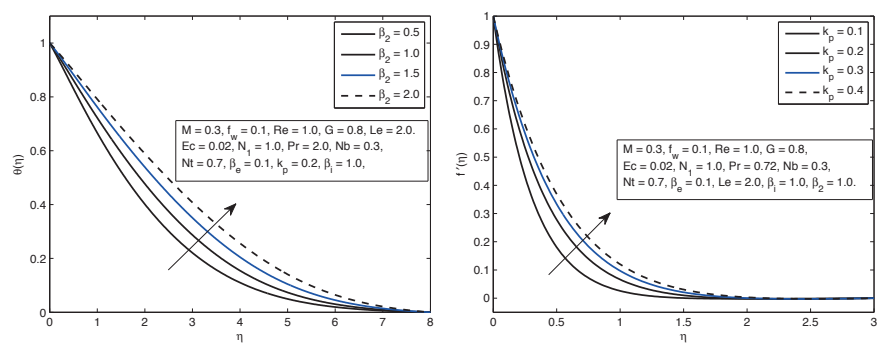

Fig. 3. Influence of $\beta_{2}$ on $\theta$ and $k_{p}$ on $f^{\prime}$

is an increasing function of the permeability parameter. This is because of the fact that the permeability parameter increases the thickness of the boundary layer of temperature and concentration. It is also expected that flow rate increases with an increase of the permeability parameter, since the holes of the porous medium become large and the resistance in fluid flow may be reduced.

Fig. 4 shows the effect of ion-slip parameter $\beta_{i}$ on horizontal and lateral velocities, respectively. In the presence
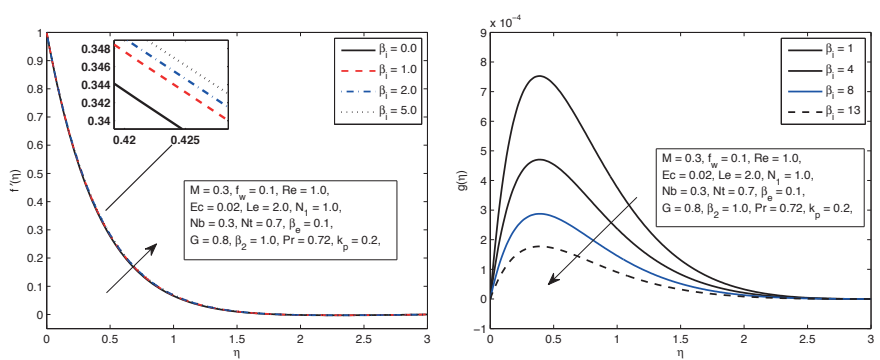

Fig. 4. Influence of $\beta_{i}$ on $f^{\prime}$ and $g$
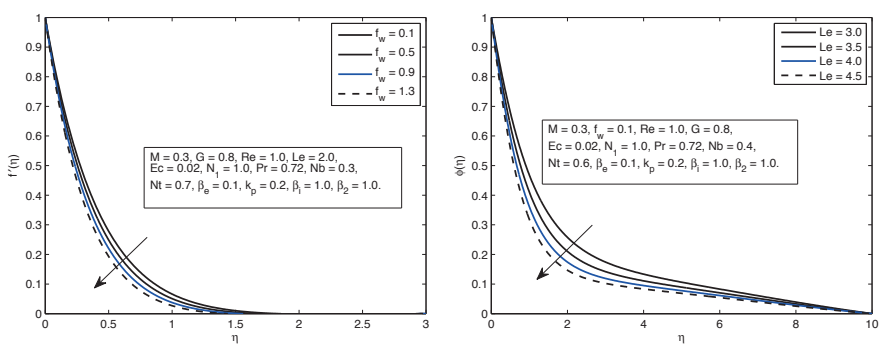

Fig. 5. Influence of $f_{w}$ on $f^{\prime}$ and Le $\phi$

of Hall and ion-slip parameters, the velocity of the flow increases, and consequently, the boundary layer thickness increases. Hence, the horizontal velocity $f^{\prime}(\eta)$ increases with the increment of $\beta_{i}$. An opposite behavior is observed for the velocity across the plate, as it decreases with the enhancement of the ion-slip parameter. Fig. 5 shows the variation of mass transfer parameter $f_{w}$ on velocity with suction (if $f_{w}>0$ ) and injection (if $f_{w}<0$ ). It is noticed that the boundary layer thickness decreases with the increase in $f_{w}$. The second figure shows the reverse relation between the nanoparticle concentration profile $\phi(\eta)$ and Lewis number $L e$, as the concentration decreases with an increase in Lewis number.

Fig. 6 depicts the typical profiles of tangential and lateral velocities for magnetic parameter $M$. By increasing the magnetic parameter $M$, a drag force, known as Lorentz force, also increases, which consequently reduces the velocity of the fluid. Since we are considering an electrically conducting micropolar nanofluid with the strong magnetic field in the direction normal to the flow, an increase in magnetic field is increasing the force in the $z$ direction, which resultantly increases the lateral velocity $g(\eta)$. To see the variation in temperature against an increasing value of Prandtl number Pr, Fig. 7 is plotted. It is observed that
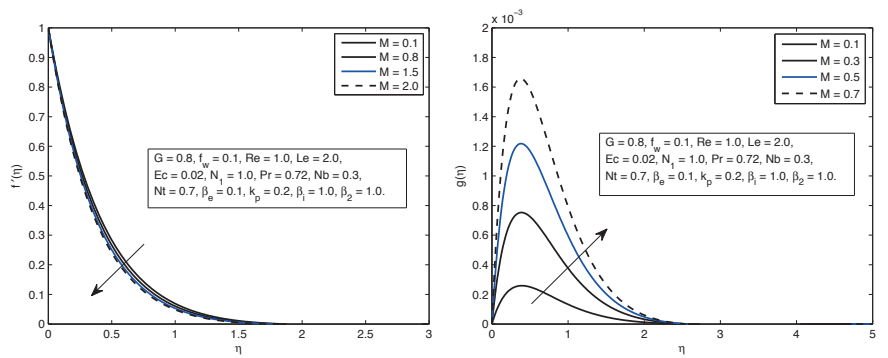

Fig. 6. Influence of $M$ on $f^{\prime}$ and $g$
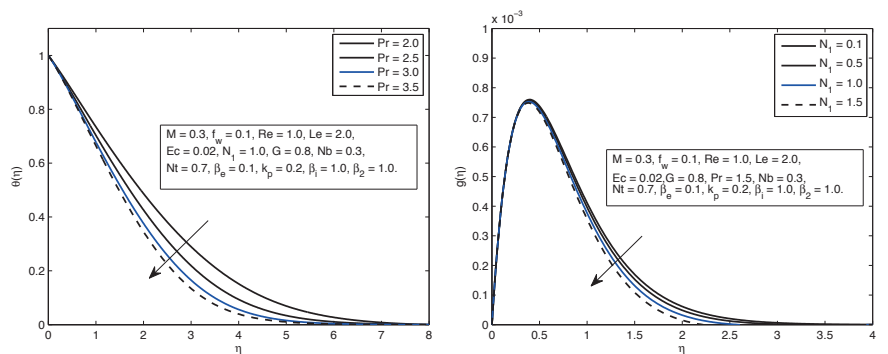

Fig. 7. Influence of $\operatorname{Pr}$ on $\theta$ and $N_{1}$ on $g$ 
for an increasing value of Prandtl number, there is a smaller temperature boundary layer thickness. Fluids having a larger Prandtl number have lower thermal diffusivity, and hence, the temperature decreases. The coupling parameter or material parameter $N_{1}$ has decreasing effects on velocity component, as the induced velocity $g(\eta)$ decreases with the increasing value of $N_{1}$, as shown in Fig. 7. The influence of Brownian motion parameter $\mathrm{Nb}$ on the temperature and concentration profile is studied in Fig. 8. From these figures, we notice that an enhancement in the values of $\mathrm{Nb}$ gives raises the temperature, while it decreases the nanoparticle concentration profile. Brownian motion is the random motion of nanoparticles suspended in a fluid, caused by the collision of particles with the fluid particles. Due to the increment in Brownian motion effect, the kinetic energy of the molecules increases and hence, the temperature increases.
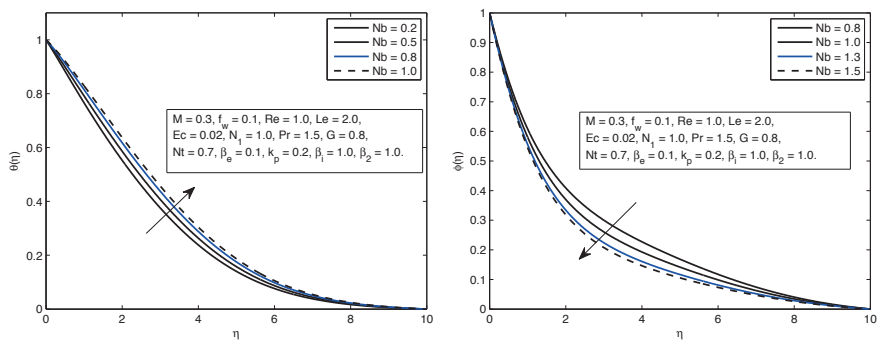

Fig. 8. Influence of $N b$ on $\theta$ and $\phi$
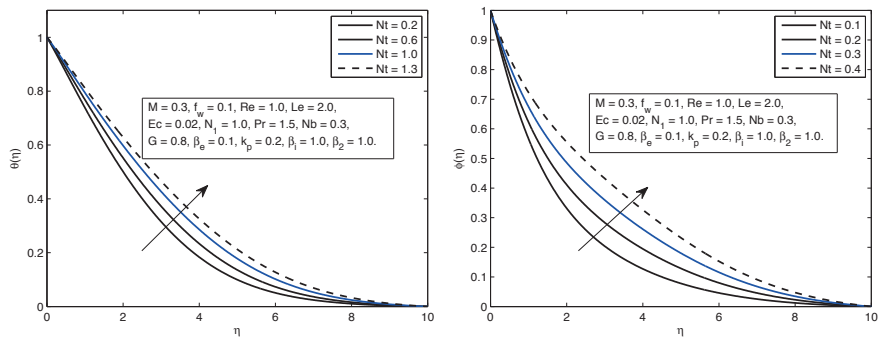

Fig. 9. Influence of $N t$ on $\theta$ and $\phi$

Fig. 9 illustrates the effect of the thermophoresis parameter $N t$ on temperature and nanoparticle concentration profiles. One can observe that temperature and concentration fields increase with the enhancement of $N t$. The thermophoresis parameter plays an important role in temperature flow. Thermophoresis force increases when $N t$ is increased, which tends to move the nanoparticles from hot region to cold region and as a result, the temperature and boundary layer thickness increases.

\section{Concluding remarks}

In this article, the effect of Hall current and ion-slip effects with variable thermal diffusivity on magneto-micropolar nanofluid on a stretching porous medium is numerically and graphically analyzed using the shooting method, which has been frequently used to calculate the solutions in many fluid mechanics prob- lems and related fields. The main points are summarized as follows:

- Thermal and concentration boundary layer thickness increases with the increase in thermophoresis parameter.

- Brownian motion parameter has opposite effect on temperature and concentration fields.

- Stronger magnetic parameter $M$ results in an increase in temperature and concentration, and a decrease in stream and lateral velocity.

- Velocity components such as stream velocity, velocity distribution along the stretching sheet, and angular velocity are all increased by the enhancement of the permeability parameter.

- A minor increase of the velocity along the $\mathrm{x}$ direction is observed for the increasing values of ion-slip and Hall current parameter.

Acknowledgements. This work was partially supported by the Higher Education Commission Pakistan.

\section{REFERENCES}

[1] S.U.S. Choi, "Enhancing thermal conductivity of fluids with nanoparticles", in Developments and Applications of Non-Newtonian Flows, pp. 99-105, eds. D.A. Siginer and H.P. Wang, ASME, New York, 1995.

[2] S.M.S. Murshed, K.C. Leong, and C. Yang, "Thermophysical and electrokinetic properties of nanofluids - a critical review", Appl. Therm. Engr. 28 (17-18), 2109-2125 (2008).

[3] S. Ozerinc, S. Kakac, and A. Yazicioglu, "Enhanced thermal conductivity of nanofluids: a state-of-the-art review", Microfluid Nanofluid 8 (2), 145-170 (2010).

[4] S.U.S. Choi, "Nanofluids: from vision to reality through research", J. Heat Transf. 131 (3), 033106-033106-9 (2009).

[5] K.V. Wong and O. Leon, "Applications of nanofluids: current and future", Adv. Mech. Engr. 2010, 1-11 (2010).

[6] J. Buongiorno, "Convective transport in nanofluids", ASME J. Heat Transf. 128 (3), 240-250 (2006).

[7] S. Das, S. Chakraborty, R.N. Jana, and O.D. Makinde, "Entropy analysis of unsteady magneto-nanofluid flow past accelerating stretching sheet with convective boundary condition", J. Appl. Math. Mech. 36 (12), 1593-1610 (2015).

[8] S.A. Shehzad, F.M. Abbasi, T. Hayat, and B. Ahmad, "Doubly stratified mixed convection flow of Maxwell nanofluid with heat generation/absorption", J. Magn. Magn. Mater. 404, 159-165 (2016).

[9] C.A. Reddy and B. Shankar, "Magneto hydrodynamics stagnation point flow of a nanofluid over an exponentially stretching sheet with an effect of chemical reaction, heat source and suction/injunction", World J. Mech. 5 (11), 211-221 (2015).

[10] S. Mansur, A. Ishak, and I. Pop, "Magnetohydrodynamic stagnation point flow of a nanofluid over a stretching/shrinking sheet with suction", PLOS ONE 10 (3), e0117733 (2015).

[11] R. Patel, "Effective viscosity of magnetic nanofluids through capillaries", Phys. Rev. E 85 (2), 026316 (2012).

[12] D. Yadav and J. Lee, "The onset of MHD nanofluid convection with Hall current effect", EPJ Plus 130 (8), Art. No. 162 (2015).

[13] M. Sheikholeslami, H.R. Ashorynejad, and P. Rana, "Lattice Boltzmann simulation of nanofluid heat transfer enhancement and entropy generation", J. Mol. Liq. 214, 86-95 (2016). 
[14] M. Sheikholeslami, S. Soleimani, and D.D. Ganji, "Effect of electric field on hydrothermal behavior of nanofluid in a complex geometry", J. Mol. Liq. 213, 153-161 (2016).

[15] M. Sheikholeslami, M.M. Rashidi, T. Hayat, and D.D. Ganji, "Free convection of magnetic nanofluid considering MFD viscosity effect", J. Mol. Liq. 218, 393-399 (2016).

[16] E. Sadeghinezhad, M. Mehrali, R. Saidur, M. Mehrali, S.T. Latibari, A.R. Akhiani, and H.S.C. Metselaar, "A comprehensive review on graphene nanofluids: recent research, development and applications", Energy Con. Mang. 111, 466-487 (2016).

[17] S. Kakac and A. Pramuanjaroenkij, "Single-phase and two-phase treatments of convective heat transfer enhancement with nanofluids - A state-of-the-art review", Int. J. Ther. Sci. 100, 75-97 (2016).

[18] H.A. Attia, "Effect of the ion slip on the MHD flow of a dusty fluid with heat transfer under exponential decaying pressure gradient", Centr. Eur. J. Phys. 3 (4), 484-507 (2005).

[19] K. Cramer and S. Pai, Magnetofluid Dynamics for Engineers and Applied Physicists, McGraw-Hill, New York, 1973.

[20] H.A. Attia, W. Abbas, and M.A.M. Abdeen, "Ion slip effect on unsteady Couette flow of a dusty fluid in the presence of uniform suction and injection with heat transfer", J. Braz. Soc. Mech. Sci. Eng. 38 (8), 2381-2391 (2015).

[21] N.N. Anika, M. Hoque, S.I. Hossain, and M. Alam, "Therma diffusion effect on unsteady viscous MHD micropolar fluid flow through an infinite vertical plate with Hall and ion-slip current", Proc. Engr. 105, 160-166 (2015).

[22] Z. Uddin and M. Kumar, "Hall and ion-slip effect on MHD boundary layer flow of a micro polar fluid past a wedge", $S c i$ entia Iranica B 20 (3), 467-476 (2013).

[23] S.S. Motsa and S. Shatery, "The effects of chemical reaction, Hall and ion-slip currents on MHD micropolar fluid flow with thermal diffusivity using a noval numerical technique", J. Appl. Math. 2012, Art. ID 689015 (2012).

[24] A.C. Eringen, "Theory of micropolar fluids", J. Math. Mech. 16 (1), 1-18 (1966).

[25] A.C. Eringen, "Theory of thermomicropolar fluids", J. Math. Anal. Appl. 38 (2), 480-496 (1972).

[26] G. Lukaszewicz, Micropolar Fluids: Theory and Applications, Birkhauser, Basel, 1999.

[27] A.C. Eringen, Microcontinuum Field Theories. Fluent Media, Springer, New York, 2001.

[28] N.A.A. Latiff, J. Uddin, O.A. Beg, and A.I. Ismail, "Unsteady forced bioconvection slip flow of a micropolar nanofluid from a stretching/shrinking sheet", Journal of Nanomaterials, Nanoengineering, and Nanosystems 230 (4), 177-187 (2016).

[29] S.E. Ahmed, M.A. Mansour, A.K. Hussein, and S. Sivasankaran, "Mixed convection from a discrete heat source in enclosures with two adjacent moving walls and filled with micropolar nanofluids", Engr. Sci. Tech., Int. J. 19 (1), 364-376(2016).

[30] P.V.S. Narayana, B. Venkateswarlu and S. Venkataramana, "Effects of Hall current and radiation absorption on MHD micropolar fluid in a rotating system", Ain Shams Engr. J. 4, 843-854 (2013).
[31] R.U. Haq, S. Nadeem, N.S. Akbar and Z.H. Khan, "Buoyancy and radiation effect on stagnation point flow of micropolar nanofluid along a vertically convective stretching surface", IEEE Trans. Nanotech. 14 (1), 42-50 (2015).

[32] N.S. Elgazery, "The effects of chemical reaction, Hall and ion slip currents on MHD flow with temperature dependent viscosity and thermal diffusivity", Comm. Nonlinear Sci. Num. Sim. 14 (4), 1267-1283 (2009).

[33] T.Y. Na, Computational Methods in Engineering Boundary Value Problems, Academic Press, 1979.

[34] M. Awais, T. Hayat, S. Irum and A. Alsaedi, "Heat generation/ absorption effects in a boundary layer stretched flow of Maxwell nanofluid: Analytic and numeric solutions", PLOS ONE 10 (6), e0129814 (2015).

[35] J. A. Khan, M. Mustafa,T. Hayat and A. Alsaedi, "Numerical study of Cattaneo-Christov heat flux model for viscoelastic flow due to an exponentially stretching surface", PLOS ONE 10 (9), e0137363 (2015)

[36] M. Mustafa, A. Mushtaq, T. Hayat and B. Ahmad, "Nonlinear radiation heat transfer effects in the natural convective boundary layer flow of nanofluid past a vertical plate: A numerical study", PLOS ONE 9 (9), e103946 (2014).

[37] A. Mushtaq, M. Mustafa, T. Hayat and A. Alsaedi, "Numerical study of the non-linear radiation heat transfer problem for the flow of a second-grade fluid", Bulg. Chem. Comm. 47 (2), 725-732 (2015).

[38] B.I. Olajuwon, "Convection heat and mass transfer in a hydromagnetic flow of a second-grade fluid in the presence of thermal radiation and thermal diffusion", Int. Comm. Heat and Mass Trans. 38 (3), 377-382 (2011).

[39] L.F Shampine, M.W. Reichelt and J. Kierzenka, "Solving boundary value problems for ordinary differential equations in MATLAB with bvp4c", http://www.mathworks.com/bvp_tutorial.

[40] S. Mansur, A. Ishak and I. Pop, "The magnetohydrodynamic stagnation point flow of a nanofluid over a stretching/shrinking sheet with suction", PLOS ONE 10 (3), e0117733 (2015).

[41] L. Cao, X. Si and L. Zheng, "Convection of Maxwell fluid over stretching porous surface with heat source/sink in presence of nanoparticles: Lie group analysis", Appl. Math. Mech. 37 (4), 433-442 (2016).

[42] J. Li, L. Zheng and L. Liu, "MHD viscoelastic flow and heat transfer over a vertical stretching sheet with Cattaneo-Christov heat flux effects", J. Mol. Liq. 221, 19-25 (2016).

[43] F.M. Abbasi and S.A. Shehzad, "Heat transfer analysis for three-dimensional flow of Maxwell fluid with temperature dependent thermal conductivity: Application of Cattaneo-Christov heat flux model", J. Mol. Liq. 220, 848-854(2016).

[44] Z. Abbas and M. Sheikh, "Numerical study of homogeneous-heterogeneous reactions on stagnation point flow of ferrofluid with non-linear slip condition", Chinese J. Chem. Engr. 25 (1), 11-17 (2017). 